\title{
OPTICAL SPECTROPHOTOMETRY OF NOVA PW VULPECULAE
}

\author{
J. Mikołajewska and M. Mikołajewski \\ Institute of Astronomy, Nicolaus Copernicus University, \\ $\mathrm{PL}-87100$ Toruh, Poland
}

PW Vul (Nowa Vul 1984 \#1) was a slow classical nova which began its outburst in July 1984 and reached optical maximum in the first week of August 1984. Eight low dispersion spectra $(160 \mathrm{~A} / \mathrm{mm})$ in the blue spectral range $(\lambda \lambda 3400-5100$ A) were secured between October 1984 and July 1985, with the ccs spectrograph equipped with 3.5 arcsec aperture image slicer and mounted on $90 \mathrm{~cm}$ schmidt-Cassegrain telescope at Torun Observatory. Kodak lla-0 plates were used. In addition two moderate dispersion $(40 \mathrm{~A} / \mathrm{mm})$ spectra in the $H_{y}-H_{B}$ range were obtained on 28 october and 3 November 1984, using the same instrumentation. Standard stars were observed on each night to remove the influence of spectral sensitivity of the photographic emulssion and atmospheric extinction. The calibration of the absolute flux scale was performed by comparing synthetic $B$ magnitudes calculated from our spectra with published B photometry of PW Vul (Voloshina 1985, Noskova et al. 1985, Kolotilov and Noskova 1986). The accuracy of the flux calibration is \pm 0.1 mag.

The emission lines were analysed with a gaussian fitting program. Blended lines were fitted with two or three gaussians only if the parameters for each profile (height and width) could be determined with confidence. Resulting fluxes of the strong lines have accuracy of about 15\%, while those of the weaker lines are less accurate $(\sim 25 \div 30 \%)$.

The calibrated low resolution spectra of $P W$ Vul are shown in Figure 1. The initial spectra were obtained when the nova was about 2 mag below optical maximum. strong $\mathrm{HI}$ Balmer emission lines with pronounced blueshifted absorption features dominate these spectra. Numerous Fell emissions are aiso visible and there is some evidence for $P$ Cygni profiles in the strongest lines. The line profiles of $H_{B}$ derived from our moderate dispersion spectra are shown in Figure 2 . Their structure is very complex with a broad emission feature and three absorption components at $V_{r} \sim-700,-1000$ and $-1500 \mathrm{~km} / \mathrm{s}$. These $P$ Cyg profiles were vanishing with time, but very weak absorption features were visible until at least 14 November 1984. Between october and November 1984 the high ionization lines of NIII and Heil developed into strong emission features. In November and December the flux in NIII $\lambda 4640$ blend was comparable with those of $H_{y}$ and $H_{\delta}$. The fluxes of most emission lines reacheo maximum values in November. Then, a gradual decline of permitted lines and development of nebular lines started. Spectra taken in 1985 are dominated by strong nebular lines from [OIII] and [Nelli].

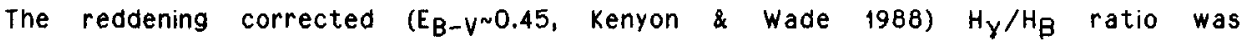
$\mathrm{H}_{y} / \mathrm{H}_{B} \approx 0.48$ during all the period of our observations is close to the value expected from case $B$ recombination, although the $H_{\alpha} / H_{B}$ flux ratio would provide a better comparison. The $H_{\alpha}$ flux observed by kenyon and Wade (1988) on 9 october 1984 combined with our observations of $\mathrm{H}_{B}$ flux on 7 and 8 october 1984, gave a reddening corrected ratio of $H_{\alpha} / H_{\beta} \approx 4.2$, which is slightly larger than allowed by case $B$ and close to the $H_{\alpha} / H_{B} \approx 4$ derived for June 1985 by Kenyon and Wade. The nearly normal 


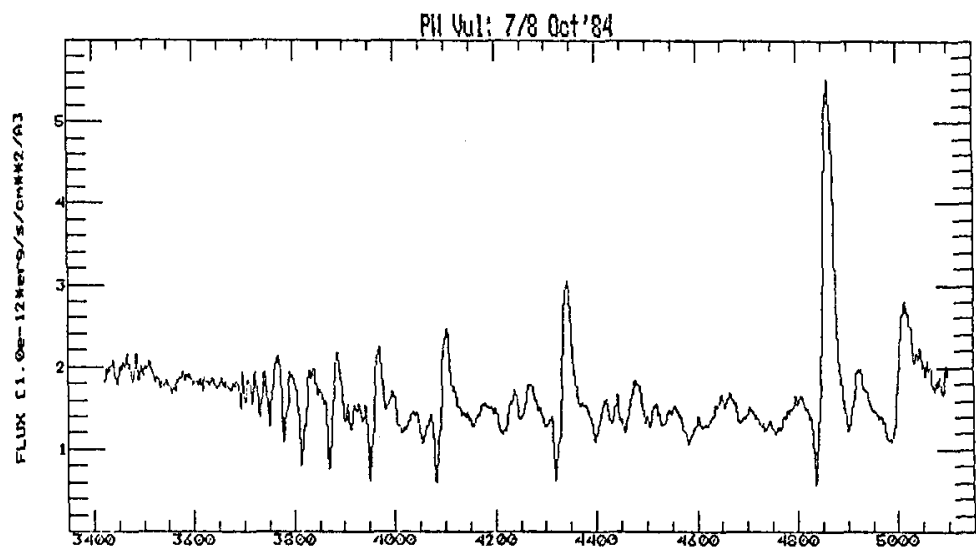

Figure 1. The low resolution spectra of PW Vul.

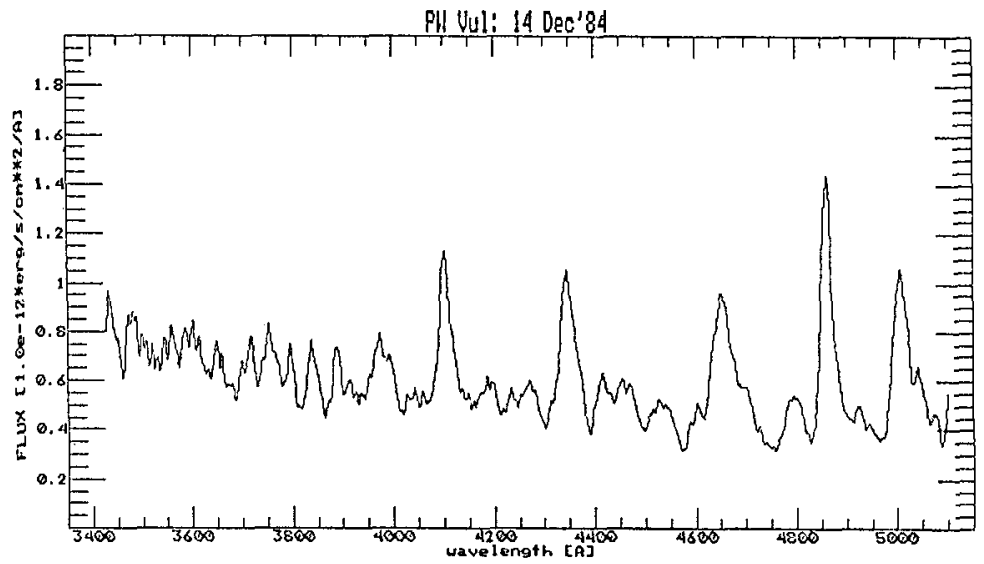

and constant $\mathrm{H}_{\alpha} / \mathrm{H}_{B}$ suggests that self-absorption was not large during disscused period.

The Hell4686/HB and Hell4686/Hel4471 flux ratios increase with time, indicating that the ionization of the nova shell increases as the radiation field of the central object shifts into the ultrafiolet. We used these ratios for estimates of the central object temperature. The derived temperatures varied from $\sim 80000 \mathrm{~K}$ in the end of October 1984 to $\sim 150000 \mathrm{~K}$ in July 1985. Assuming that the strong emission lines are produced in a nebula photoionized by a single temperature blackbody, and

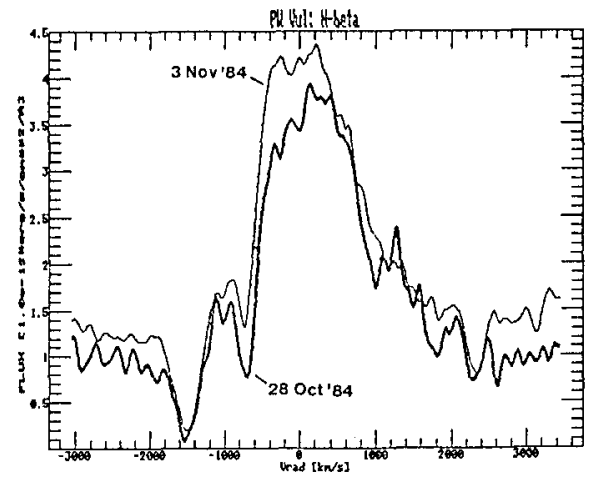

Figure 2. The line profiles of $\mathrm{H}_{B}$ using the reddening corrected $\mathrm{H}_{B}$ and Hell 4686 fluxes, we found that the luminosity of the central object was $\sim 4000 \mathrm{~L}_{0}$ in october and November 1984, then declined to $1200 \mathrm{~L}_{0}$ in December 1984 and to $2200 \mathrm{~L}_{0}$ in July 1985.

References:

Kenyon, S.J., Wade, R.A., 1988, P.A.S.P., 98, 935.

Kolotilov, E.A., Noskova, R.l, 1986, Pisma $v$ Astr. Zhu., 12, 883.

Noskova, R.I., Zaitseva, G.V., Kolotilov, E.A,, 1985, Pisma $v$ Astr. Zhu., 11, 613. Voloshina, I., 1985, Astr. Tsirk. No. 1358, 4 